\title{
Identification of Unknown Filter in a Half-Strip
}

\author{
Volodymyr Dilnyi ${ }^{1,2}$. Khrystyna Huk ${ }^{2}$
}

Received: 9 October 2017 / Accepted: 18 March 2019 / Published online: 26 March 2019

(C) The Author(s) 2019

\begin{abstract}
We consider the analogue of the classic Wiener filtering theory to a half-strip of complex domain. In this paper all test signal obtained that solving the filter identification problem for the Hardy spaces in a half-strip. This result can be used for the investigations of electrical, optical, acoustical signals.
\end{abstract}

Keywords Signal processing · Amplitude spectrum · Convolution · Fourier transform · Hardy spaces · Filter

\section{Introduction}

In Wiener's mathematical filtering theory [1] a signal is a function of the continuous $(t \in \gamma$, $\gamma: R \rightarrow C$ ) time parameter $t$ and a filter $\Phi$ is a device ("a box") transforming an input signal into a certain output signal, $g \rightarrow \Phi g$. The energy of a signal $g$ is proportional to

$$
\int_{\gamma}|g(z)|^{2}|d z| .
$$

Without entering into the physical nature (optical, electrical) of a stationary filter $\Phi$, we consider it as a translation invariant linear operator on the corresponding $L^{2}$ space.

The function $G=\mathcal{F}^{-1} g$, where $g \rightarrow \mathcal{F}^{-1} g$ is the inverse Fourier transforms, represents what is called the amplitude spectrum of $g$.

\footnotetext{
$凶$ K. Huk

khrystyna.huk2711@gmail.com

V. Dilnyi

dilnyiv@gmail.com

1 Institute of Mathematics, Cracow University of Technology, Warszawska str., 24, 31-155, Cracow, Poland

2 Educational and Scientific Institute of Physics, Mathematics, Economics and Innovative Technologies, Drohobych Ivan Franco State Pedagogical University, Stryiska Street, 3, Drohobych, 82100, Ukraine
} 
The following are among the major problem of signal processing:

determine an unknown filter $\Phi: g \rightarrow \psi$ ("black box") from an analysis of $g$ and $\psi$; in particular, reconstruct, if possible, a filter knowing the energy densities $\left|\mathcal{F}^{-1} g\right|^{2},\left|\mathcal{F}^{-1} f\right|^{2}$ of an input-output pair.

The last problem is a variation of M. Kac' famous question "Can one hear the shape of a drum?" [2]. Above and similar problems are studied by N. Wiener, D. Newman, B. Nyman, A. Beurling, H. Reinhard, P. Masani [3].

\section{The Half-Strip Case}

The complex domain provides a natural processing framework for signals with intensity and direction components (see [4-6]).

We consider the above problem for the case of an unknown filter $f$ on the half-strip $D_{\sigma}=\{z:|\Im z|<\sigma, \Re z<0\}, \sigma>0$. The aim of this paper is to construct all detecting signals $g$ on $D_{\sigma}^{*}=\mathbb{C} \backslash \bar{D}_{\sigma}$ under some natural conditions.

The circle of ideas surrounding signal processing and Fourier transform has a long history, and has found a number applications. J. Martinez, R. Heusdens and R. Hendriks [7] investigate the characteristics of the signal and the connections of the generalized Fourier transform to analyticity. E. Sejdic, I. Djurovic and L. Stankocic relate the Fractional Fourier transform to other mathematical transforms and discuss various approaches for practical realizations of this transform [8].

Let $E^{p}\left[D_{\sigma}\right]$ and $E^{p}\left[D_{\sigma}^{*}\right], 1 \leq p<+\infty, \sigma>0$, be the (Hardy) spaces of holomorphic functions respectively in the domains $D_{\sigma}$ and $D_{\sigma}^{*}$, for which

$$
\|f\|:=\sup \left\{\int_{\mu}|f(z)|^{p}|d z|\right\}^{1 / p}<+\infty,
$$

where supremum is taken over all segments $\mu$, that are contained in $D_{\sigma}$ and $D_{\sigma}^{*}$ respectively. The amplitude spectrum of a signal $g \in E^{p}\left[D_{\sigma}^{*}\right]$ is defined by the formula

$$
G(z)=\frac{1}{i \sqrt{2 \pi}} \int_{\partial D_{\sigma}} g(w) e^{z w} d w .
$$

Obviously, the above equality is a natural analogue of the Fourier transform for the half-strip.

The filter identification problem for the half-strip is to find, if possible, a test signal $g \in E^{2}\left[D_{\sigma}^{*}\right]$ whose output

$$
f * g(\tau)=\int_{\partial D_{\sigma}} g(w) f(w+\tau) d w
$$

measured at all time moments $\tau \leq 0$ defines uniquely an unknown filter $f \in E^{2}\left[D_{\sigma}\right]$. More precisely, the question is whether there exists $g \in E_{*}^{2}\left[D_{\sigma}\right]$ such that $g * f(\tau)=0$ for all $\tau \leq 0$ implies $f \equiv 0$ ?

Let $H^{p}\left(\mathbb{C}_{+}\right), 1 \leq p<+\infty$, be the Hardy space of holomorphic in $\mathbb{C}_{+}=\{z: \Re z>0\}$ functions $f$, for which

$$
\|f\|^{p}=\sup _{x>0}\left\{\int_{-\infty}^{+\infty}|f(x+i y)|^{p} d y\right\}<+\infty .
$$


Theorem $\mathbf{S}$ [9] The space $H^{p}\left(\mathbb{C}_{+}\right), 1 \leq p<+\infty$, coincides with the space of holomorphic in $\mathbb{C}_{+}$functions $f$, for which

$$
\|f\|_{*}^{p}=\sup _{\varphi \in\left(-\frac{\pi}{2} ; \frac{\pi}{2}\right)}\left\{\int_{0}^{+\infty}\left|f\left(r e^{i \varphi}\right)\right|^{p} d r\right\}<+\infty .
$$

Let $H_{\sigma}^{p}\left(\mathbb{C}_{+}\right)$be a space of holomorphic in $\mathbb{C}_{+}$functions, for which

$$
\|g\|:=\sup _{\varphi \in\left(-\frac{\pi}{2} ; \frac{\pi}{2}\right)}\left\{\int_{0}^{+\infty}\left|g\left(r e^{i \varphi}\right)\right|^{p} e^{-p \sigma r|\sin \varphi|} d r\right\}^{1 / p}<+\infty .
$$

Formula (1) establish the bijections of $E^{2}\left[D_{\sigma}^{*}\right]$ onto $H_{\sigma}^{2}\left(\mathbb{C}_{+}\right)$and inverse formula

$$
g(w)=\frac{1}{\sqrt{2 \pi}} \int_{0}^{+\infty} G(x) e^{-x w} d x, \quad \Re w>0
$$

holds.

\section{The Main Result}

Theorem 1. Let the amplitude spectrum $G$ of a signal $g \in E^{2}\left[D_{\sigma}^{*}\right]$ be continuous and zerofree in $\{z: \Re z \geq 0\}, f \in E^{2}\left[D_{\sigma}\right]$. Then

$$
(\forall \tau \leq 0) \quad f * g(\tau)=0
$$

implies $f \equiv 0$ if and only if one of the following conditions holds:

a) $g$ admits a holomorphic continuation as an entire function and

$$
(\forall c \in \mathbb{R}): g(w) \exp \left(-c e^{-\frac{w \pi}{2 \sigma}}\right) \notin E^{2}\left[D_{\sigma}\right] ;
$$

b) $g$ does not admit an analytic continuation as an entire function.

Proof The integral boundary function $h: \mathbb{R} \rightarrow \mathbb{R}$ of a function $G \in H_{\sigma}^{p}\left(\mathbb{C}_{+}\right)$is defined [10] up to an additive constant at points of continuity by the equality [10]

$$
h\left(t_{2}\right)-h\left(t_{1}\right)=\lim _{x \rightarrow 0+} \int_{t_{1}}^{t_{2}} \ln |G(x+i y)| d y-\int_{t_{1}}^{t_{2}} \ln |G(i y)| d y .
$$

Since $G$ is continuous on $\overline{\mathbb{C}}_{+}$and $G(z) \neq 0$ for all $z \in \overline{\mathbb{C}}_{+}$, we have $|\ln | G(z)|| \leq M_{R}$ for $z \in\{z: \Re z \geq 0,|z| \leq R\}$. Therefore by Fatou's lemma $h \equiv$ const. Since $G$ is zero-free in $\mathbb{C}_{+}$, by the criterion of solvability $[11,12]$ equation (3) has only trivial (zero) solution iff

$$
\limsup _{x \rightarrow+\infty}\left(\frac{\ln |G(x)|}{x}+\frac{2 \sigma}{\pi} \ln x\right)=+\infty
$$


We will show that (5) implies condition a) or b). Consider the two alternatives:

$$
\text { 1) } \lim _{x \rightarrow+\infty} \frac{\ln |G(x)|}{x}=-\infty
$$

From the last formula we obtain that the integral on the right hand side of (2) converges uniformly on any compact subset of $\mathbb{C}_{+}$, hence $g$ is an entire function.

We suppose the contrary to (4). Since $g$ is an analytic function in an each closed rectangle $\bar{M}_{k}, k<0$, where $M_{k}=\left\{z: z \in D_{\sigma}, \Re z>k\right\}$. By Cauchy formula we obtain

$$
\int_{\partial M_{k}} g(w) e^{z w} d w=0, \quad k<0,
$$

then by (1) we have

$$
G(z)=\frac{1}{i \sqrt{2 \pi}} \int_{\partial D_{\sigma} \backslash \bar{M}_{k}} g(w) e^{z w} d w .
$$

Let $q(w):=g(w) \exp \left(-c e^{-\frac{w \sigma}{2 \sigma}}\right) \in E^{2}\left[D_{\sigma}\right]$ for some $c>0$.

Hence for $k<0$ we obtain

$$
\begin{aligned}
|G(x)|= & \frac{1}{\sqrt{2 \pi}}\left|\int_{\partial\left(D_{\sigma} \backslash \bar{M}_{k}\right)} q(w) \exp \left(c e^{-\frac{w \pi}{2 \sigma}}\right) e^{w x} d w\right| \\
\leq & \frac{1}{\sqrt{2 \pi}} \int_{\partial\left(D_{\sigma} \backslash \bar{M}_{k}\right)}|q(w)| \exp \left(c e^{-\frac{u \pi}{2 \sigma}} \cos \frac{v \pi}{2 \sigma}\right) e^{u x}|d w| \\
= & \frac{1}{\sqrt{2 \pi}}\left(\int_{-\infty}^{k}|q(u-i \sigma)| e^{u x} d u+\int_{-\sigma}^{\sigma}|q(k+i v)| \exp \left(c e^{-\frac{k \pi}{2 \sigma}} \cos \frac{v \pi}{2 \sigma}\right) e^{k x} d v\right. \\
& \left.+\int_{-\infty}^{k}|q(u+i \sigma)| e^{u x} d u\right) \\
\leq & \frac{1}{\sqrt{2 \pi}}\left(\frac{e^{k x}}{\sqrt{2 x}}\left(\int_{-\infty}^{0}|q(u-i \sigma)|^{2} d u\right)^{1 / 2}+\exp \left(c e^{-\frac{k \pi}{2 \sigma}}\right) e^{k x} \sqrt{2 \sigma}\right. \\
& \left.\cdot\left(\int_{-\sigma}^{\sigma}|q(k+i v)|^{2} d v\right)^{1 / 2}+\frac{e^{k x}}{\sqrt{2 x}}\left(\int_{-\infty}^{0}|q(u+i \sigma)|^{2} d u\right)^{1 / 2}\right) .
\end{aligned}
$$

The last inequality is a consequence of Schwarz inequality. If $k=-\frac{2 \sigma}{\pi} \ln x$, then

$$
\begin{aligned}
|G(x)| & \leq \frac{c_{7}}{\sqrt{x}} \exp \left(-\frac{2 \sigma}{\pi} x \ln x\right)+c_{8} e^{c x} \exp \left(-\frac{2 \sigma}{\pi} x \ln x\right) \\
& \leq c_{9} e^{c x} \exp \left(-\frac{2 \sigma}{\pi} x \ln x\right), \quad x>1 .
\end{aligned}
$$


Let $\psi(z):=G(z) e^{-c z} \exp \left(-\frac{2 \sigma}{\pi} z \ln z\right)$. Obviously $\psi \in L^{2}\left(\partial \mathbb{C}_{+}\right)$and for all $\varepsilon>0$ we have $\psi(x) e^{-\varepsilon x} \in L^{2}(0 ;+\infty)$ and by equality (1) we obtain $G \in H_{\sigma}^{2}\left(\mathbb{C}_{+}\right)$, hence for all $\gamma \in(1 ; 2]$

$$
(\forall \varepsilon>0): \sup _{|\varphi|<\frac{\pi}{2}}\left\{\int_{0}^{+\infty}\left|\psi\left(r e^{i \varphi}\right)\right|^{2} \exp \left(-\varepsilon r e^{\gamma}\right) d r\right\}<+\infty
$$

From a Phragmen-Lindelof type theorem for the half-plane [13], we obtain $\psi \in H^{2}\left(\mathbb{C}_{+}\right)$. It means

$$
G(z) e^{c z} \exp \left(\frac{2 \sigma}{\pi} z \ln z\right) \in H^{2}\left(\mathbb{C}_{+}\right)
$$

and

$$
\left|G(z) \exp \left\{\frac{2 \sigma}{\pi} z \ln z\right\}\right| \leqslant \frac{e^{c x}}{\sqrt[p]{x}} .
$$

Therefore $\ln |G(x)|+\frac{2 \sigma}{\pi} x \ln x \leqslant c x$, if $x \geq 1$, since

$$
\limsup _{x \rightarrow+\infty}\left(\frac{\ln |G(x)|}{x}+\frac{2 \sigma}{\pi} \ln x\right)<+\infty .
$$

This is a contradiction.

The second alternative is

$$
\text { 2) } \limsup _{x \rightarrow+\infty} \frac{\ln |G(x)|}{x}>-\infty \text {. }
$$

Let $g \in E_{*}^{2}\left[D_{\sigma}\right]$ be an entire function. Then $g$ is an analytic function in each closed rectangle $\bar{M}_{k}, k<0$, where $M_{k}=\left\{z: z \in D_{\sigma}, \Re z>k\right\}$. By the Cauchy formula we obtain

$$
\int_{\partial M_{k}} g(w) e^{z w} d w=0, \quad k<0,
$$

then by (7) we obtain

$$
|G(x)|=\frac{1}{\sqrt{2 \pi}} \int_{\partial D_{\sigma} \backslash \bar{M}_{k}}|g(w)| e^{x u}|d w|=\frac{1}{\sqrt{2 \pi}}\left(I_{1}+I_{2}+I_{3}\right), \quad z=x+i y, w=u+i v,
$$

for $x>0$. Then, by Schwarz inequality

$$
\begin{aligned}
I_{1} & =\int_{-\infty}^{k}|g(u-i \sigma)| e^{x u} d u \leq\left(\int_{-\infty}^{k}|g(u-i \sigma)|^{2} d u \cdot \int_{-\infty}^{k} e^{2 x u}\right)^{1 / 2} d u \\
& \leq\left(\int_{-\infty}^{0}|g(u-i \sigma)|^{2} d u \cdot \frac{e^{2 x k}}{2 x}\right)^{1 / 2} \leq c_{2} \frac{e^{x k}}{\sqrt{x}},
\end{aligned}
$$


analogously

$$
I_{3}=\int_{-\infty}^{k}|g(u+i \sigma)| e^{x u} d u \leq c_{3} \frac{e^{x k}}{\sqrt{x}} .
$$

Further

$$
I_{2}=\int_{-\sigma}^{\sigma}|g(k+i v)| e^{x k} d v \leq \max _{v \in[-\sigma ; \sigma]}\{|g(k+i v)|\} e^{x k} \int_{-\sigma}^{\sigma} d v \leq J(k) e^{k x},
$$

where $J(k)=2 \sigma \max \{|g(t+i v)|: v \in[-\sigma ; \sigma], t \in[k ; 0]\}, k<0$.

If $\sup _{k<0}\{J(k)\}<+\infty$, then the function $g$ belongs to the Hardy spaces in both domains $D_{\sigma}$ and $D_{\sigma}^{*}$. Then $g \equiv 0$ and by (1) $G \equiv 0$ hence the theorem is proved. In the other way from the nonincreasity of $J$ we have $\lim _{k \rightarrow-\infty} J(k)=+\infty$. Let $J_{1}$ be defined on intervals of decreasing of $J$ as $J_{2}=J$. Then the inverse function $J_{1}$ of the function $-J$ increase on $(-\infty ; 0)$ and $\lim _{s \rightarrow-\infty} J_{1}(s)=-\infty$. As $k$ in (6) is an arbitrary negative number, we can choose $k=J_{1}(-x)$, then $I_{2} \leq J\left(J_{1}(-x)\right) e^{x J_{1}(-x)}=x e^{x J_{1}(-x)}$. Hence $|G(x)| \leq c_{4} x e^{x J_{1}(-x)}$, $x>1$, and we obtain

$$
\lim _{x \rightarrow+\infty} \frac{\ln |G(x)|}{x}=\lim _{x \rightarrow+\infty} J_{1}(-x)=-\infty
$$

This is a contradiction.

Conversely, b) suppose, condition (9) is not valid. Then by (6) the integral on the right hand side of (2) converges uniformly on any compact subset of $\mathbb{C}_{+}$, hence $g$ is the entire function. This is a contradiction. Condition (5) is a simple consequence of (9).

Condition a) let $G \in H_{\sigma}^{2}\left(\mathbb{C}_{+}\right)$and (4) is valid. Then by (1) equality (2) is valid for all $w \in \mathbb{C}_{+}$. Let $G_{1}(z)=G(z) \exp \left(\frac{2 \sigma}{\pi} z \ln z-c z\right) \in H^{2}\left(\mathbb{C}_{+}\right)$for some $c>0$. Then after a change of the line of integration from $\{x: x>0\}$ to $\left\{t \exp \left(-\frac{(w-c) \pi}{2 \sigma}: t>0\right)\right\}$ we obtain

$$
\begin{aligned}
|g(w)|= & \frac{1}{\sqrt{2 \pi}}\left|\int_{0}^{+\infty} G_{1}\left(t e^{-\frac{(w-c) \pi}{2 \sigma}}\right) \exp \left(-\frac{2 \sigma}{\pi} t \ln t e^{-\frac{(w-c) \pi}{2 \sigma}}\right) e^{-\frac{(w-c) \pi}{2 \sigma}} d t\right| \\
\leq & \frac{1}{\sqrt{2 \pi}}\left(\int_{0}^{+\infty}\left|G_{1}\left(t e^{-\frac{(w-c) \pi}{2 \sigma}}\right) e^{-\frac{(w-c) \pi}{2 \sigma}}\right|^{2} d t \cdot \int_{0}^{+\infty}\left|\exp \left(-\frac{4 \sigma}{\pi} t \ln t e^{-\frac{(w-c) \pi}{2 \sigma}}\right)\right|^{2} d t\right)^{1 / 2} \\
\leq & c_{10}\left(e^{-\frac{(u-c) \pi}{2 \sigma}} \exp \left(\frac{1}{2} \ln \left(\frac{4 \sigma}{\pi} e^{-\frac{(u-c) \pi}{2 \sigma}} \cos \frac{v \pi}{2 \sigma}\right)\right)\right. \\
& \left.\cdot \exp \left(\exp \left(\ln \left(\frac{4 \sigma}{\pi} e^{-\frac{(u-c) \pi}{2 \sigma}} \cos \frac{v \pi}{2 \sigma}\right)-1\right)\right)\right)^{1 / 2} .
\end{aligned}
$$

The last estimate follows from [14]. Then we have

$$
|g(w)| \leq c_{11} e^{-\frac{2 u \pi}{\sigma}} \exp \left(\frac{1}{e} \frac{2 \sigma}{\pi} e^{-\frac{(u-c) \pi}{2 \sigma}} \cos \frac{v \pi}{2 \sigma}\right) .
$$

Function $q_{1}(w)=g(w) \exp \left(-c_{12} e^{-\frac{w \pi}{2 \sigma}}\right)$, where $c_{12}=\frac{2 \sigma}{\pi e} e^{\frac{c \pi}{2 \sigma}}$, satisfy the conditions of the Phragmen-Lindelof type theorem for a half-strip (see [11]) because $g_{2} \in L^{2}\left(\partial D_{\sigma}\right)$. Then $g_{2} \in E^{2}\left[D_{\sigma}\right]$, hence condition (5) is valid for $c=c_{12}$. 
Publisher's Note Springer Nature remains neutral with regard to jurisdictional claims in published maps and institutional affiliations.

Open Access This article is distributed under the terms of the Creative Commons Attribution 4.0 International License (http://creativecommons.org/licenses/by/4.0/), which permits unrestricted use, distribution, and reproduction in any medium, provided you give appropriate credit to the original author(s) and the source, provide a link to the Creative Commons license, and indicate if changes were made.

\section{References}

1. Wiener, N.: Extrapolation, Interpolation and Smoothing of Stationary Time Series. Wiley, New York (1949)

2. Kac, M.: Can one hear the shape of a drum? Am. Math. Mon. 73(II), 1-23 (1966)

3. Nikolski, N.: Operators, Functions and Systems: An Easy Reading. Hardy, Hankel and Toeplitz. AMS, Providence (2002)

4. Mandig, D., Goh, V.: Complex Value Nonlinear Adaptive Filters. Wiley, New York (2009)

5. Haykin, S.: Adaptive Filter Theory. Prentice Hall, New York (2013)

6. Bruna, J., Massaneda, X., Ortega-Cerdà, J.: Connections between signal processing and complex analysis. Contrib. Sci. 2(3), 345-357 (2003)

7. Martinez, J., Heusdens, R., Hendriks, R.: A generalized Fourier domain: signal processing framework and applications. Signal Process. 93(5), 1259-1267 (2013)

8. Sejdic, E., Djurovic, I., Stankocic, L.: Fractional Fourier transform as a signal processing tool: an overview of recent developments. Signal Process. 91(6), 1351-1369 (2011)

9. Siedletskyi, A.: An equivalent definition of $H^{p}$ spaces in the half-plane and some applications. Mat. Sb. (N.S.) 96(138), 75-82 (1975)

10. Vynnytskyi, B.: On zeros of some classes of functions analytic in half-plane. Mat. Stud. 6, 67-72 (1996) (in Ukrainian)

11. Dilnyi, V.: On cyclic functions in weighted Hardy spaces. J. Math. Phys. Anal. Geom. 7(1), 19-33 (2011)

12. Dilnyi, V., Vynnytskyi, B.: A generalization of the Beurling-Lax theorem. Math. Notes 79(3), 335-341 (2006)

13. Koosis, P.: Introduction to $H_{p}$ Spaces. Cambridge Tracts in Mathematics, vol. 115. Cambridge University Press, Cambridge (1998)

14. Fedoryuk, M.: Asymptotics: Integrals and Sums. Nauka, Moscow (1987) (in Russian) 